\title{
A Spatially Varying Pulse Compression Filter for Coded Excitation Signals
}

\author{
Jose R. Sanchez \\ Electrical and Computer Engineering Department \\ Bradley University \\ Peoria, Illinois 61625 \\ Email: jsm@bradley.edu
}

\author{
Michael Oelze \\ Electrical and Computer Engineering Department \\ University of Illinois \\ Urbana, Illinois 61801 \\ Email: oelze@illinois.edu
}

\begin{abstract}
Coded excitation and pulse compression techniques have been used to improve the echo signal-to-noise ratio (eSNR) in ultrasonic imaging. Pulse compression can be performed using a Wiener filter. In this work, the effects of applying a spatially varying Wiener filter were evaluated. Specifically, a technique to adaptively compensate for the spatial changes in eSNR in the compression scheme was examined. Simulations and experiments were conducted with a single-element transducer (f/2.66) having a center frequency of $2.25 \mathrm{MHz}$ and a -3-dB bandwidth of $50 \%$. In simulations, tissue-mimicking phantoms with 12 point targets were imaged. In experiments, anechoic targets from the ATS 539 tissue-mimicking phantom were imaged. For simulated echoes compressed with a spatially varying Wiener filter (SVWF), an increase in eSNR of $235 \%$ was obtained over the non-SVWF at depths that typically could not be imaged using a conventional pulsed (CP) excitation. For the experiments with the anechoic targets, it was observed that the contrast-to-noise ratio was improved by $245 \%$ over CP when compressing with a SVWF. Simulation and experimental results suggest that accounting for local variations in the eSNR can lead to significant improvements in the compression performance. Specifically, the eSNR was increased in deeper areas imaged at the expense of a small degradation in spatial resolution.
\end{abstract}

\section{INTRODUCTION}

In coded excitation, the objective is to obtain a time bandwidth product (TBP) greater than unity [1]. For ultrasound transducers, the bandwidth is fixed. Consequently, the TBP can only be increased by elongating the pulse duration. An excitation signal with a longer duration than a conventional excitation signal has more energy, which results in increases of echo signal-to-noise ratio (eSNR) [2]. However, because the pulse duration is increased the axial resolution degrades. To restore the axial resolution, pulse compression techniques are used.

Pulse compression could be performed using various filtering techniques: matched filtering, inverse filtering, and Wiener filtering. A matched filter uses correlation techniques, which has excellent noise suppression capabilities. However, a matched filter will introduce axial sidelobes and decreases the axial resolution by a factor of two. An inverse filter provides the opposite scenario; it contains no sidelobes and does not deteriorate the axial resolution but at the expense of amplifying the noise in the system. A Wiener filter is described by the following expression:

$$
H_{W i e n e r}(f)=\frac{\Psi^{*}(f)}{|\Psi(f)|^{2}+\overline{e S N R^{-1}(f)}},
$$

$\overline{e S N R}$ is the echo signal-to-noise ratio per frequency channel and $\Psi(f)$ corresponds to the Fourier transform of the excitation signal. If $\overline{e S N R}$ is large then the Wiener filter equation approaches an inverse squared filter. Conversely, if the $\overline{e S N R}$ is small then the Wiener filter equation approaches a matched filter. Therefore, the Wiener filter equation can be modified as follows

$$
H_{\text {Wiener }}(f)=\frac{\Psi^{*}(f)}{|\Psi(f)|^{2}+\gamma \overline{e S N R^{-1}(f)}},
$$

where $\gamma$ is a smoothing parameter that controls the tradeoff between sidelobe levels, axial resolution, and echo signal-tonoise ratio.

The compression of a scan line using the Wiener filter described in (2) contain a constant $\overline{e S N R(f)}$. Compression on a per scan line basis can be performed but the compression performance depends on the objects being imaged. In this study, the effects of the spatially varying nature of the $\overline{e S N R}$ were evaluated. In addition, a technique to compensate for the changes in $\overline{e S N R}$ spatially in the pulse compression scheme was examined. The overall objective is to improve the compression performance (sidelobes, spatial resolution, and eSNR) throughout the image.

\section{Problem Formulation}

In an ultrasonic B-mode image, the image is constructed by collecting data from many adjacent scan lines. Estimating a single $\overline{e S N R(f)}$ for all scan lines could be problematic in various scenarios. For example, in one scenario, one scan line could have a large amount of electronic noise compared to other scan lines making up an image. In another example, a portion of a scan line could have a very strong specular reflection from a point target and the remaining portion of the scan line contain speckle from weak scatterers. Consequently, the specular reflector would increase the eSNR estimated for the scan line, which would in turn adjust the operating point of the Weiner filter. An increase in the eSNR would move the Weiner filter closer to an inverse filter. As a Wiener filter 
approaches an inverse filter the sidelobes are reduced, the axial resolution is improved and the noise is amplified. As a result, depending on the exact location of the operating point, the filter could be more efficient in compressing the strong specular reflector but could increase the noise in the portion of the signal with speckle only. The end result would be an image with poor quality. The image could partially be improved by adjusting the gamma parameter to shift the operating point towards a matched filter. In this scenario, the speckle portion of the signal could be moved above the noise floor, but the specular reflection would be characterized by larger sidelobes and a decrease in axial resolution. Therefore, to ameliorate these effects a Wiener filter that takes into account the spatially varying nature of the eSNR was studied.

The spatially varying Wiener filter (SVWF) based on a spatially varying eSNR is described by the following equation:

$$
\beta_{R E C}\left(f, x_{0}\right)=\frac{\mid \Psi(f)}{\left.\Psi(f)\right|^{2}+\gamma{\overline{e S N R_{0}}}^{-1}\left(f, x_{0}\right)},
$$

where $\overline{e S N R}_{0}$ is the echo-signal-to-noise ratio per frequency channel for the spatial region of interest defined by the axial location, $x_{0} . \overline{e S N R}_{0}$ can be described by the following expression:

$$
\overline{e S N R_{0}\left(f, x_{0}\right)}=\overline{e S N R(f, x)} \operatorname{var}\left(g\left(x_{0}\right)\right),
$$

where the variance of the echo signal $g\left(x_{0}\right)$ is taken over a windowed segment using a rectangular window centered at $x_{0}$. To obtain the variance of the echo signal at location $x_{0}$, the backscattered data are compressed twice. The first compression creates a spatial map of signal strength throughout the whole image region by assuming a uniform eSNR for the scan line. From there, a map of the variance of the compressed echoes for smaller sub-regions of the image is determined. This map is used in the SVWF to adaptively select the operating point of the Wiener filter for each particular region. Consequently, regions containing stronger signals will be filtered closer to an inverse filter to improve spatial resolution and sidelobe levels. Regions containing weaker echoes will be filtered closer to a matched filter to make targets visible by removing the noise at the expense of increasing the sidelobe levels and deteriorating the axial resolution. Compressing with the spatial map of eSNR can become significant when frequency-dependent attenuation is present because the image quality can be improved at all depths. The final image is constructed by stitching together each of the the compressed regions of interest.

\section{Methods And PRocedure}

\section{A. Coded Excitation}

The coded excitation scheme used to evaluate the filter in this study was the REC technique [3]. The REC technique was chosen because of its capabilities to improve the axial resolution of ultrasonic imaging system by a factor of two over conventional coded excitation schemes. Nonetheless, the filtering techniques studied herein can be applied to general techniques involving coded excitation and pulse compression.

\section{B. Sources and Phantoms}

Computer simulations were carried out in MATLAB (Mathworks, Natick, MA) to characterize the performance of the Wiener filter based on spatially varying maps of $\overline{e S N R}$. The simulations used the received pulse-echo pressure field model observed in [4]. The simulation parameters were made to match experimental measurements. A $2.25 \mathrm{MHz}$ singleelement transducer (f/2.66) with a fractional bandwidth of $70 \%$ at $-6 \mathrm{~dB}$ was the model for CP. For REC, the impulse response of the transducer was constructed to have double the fractional bandwidth $(150 \%)$ at $-6 \mathrm{~dB}$.

In simulations, to assess the performance of the Wiener filter a phantom containing 12 point targets spaced $5 \mathrm{~mm}$ apart in water was evaluated. Attenuation of $2 \mathrm{~dB} \mathrm{MHz}^{-1} \mathrm{~cm}^{-1}$ was added to the simulation so that a decrease in backscattered energy from the point targets away from the source was achieved. This phantom was evaluated for two different cases of noise floors: low noise and high noise. For the low noise case, the $e S N R$ for the region including the first point target was $62 \mathrm{~dB}$. For the high noise case, the $e S N R$ in the region including the first point target was $22 \mathrm{~dB}$. In experimental measurements, anechoic targets from an ATS 539 tissuemimicking phantom were imaged.

\section{Image quality metrics}

To quantify the effects of the SVWF the following image quality metrics were used:

1) Modulation transfer function (MTF): MTF [5] is an important metric for determining axial resolution of an imaging system. MTF is the magnitude of the optical transfer function and is defined by

$$
\operatorname{MTF}(k)=\left|\frac{H(k)}{H(0)}\right|,
$$

where $H(k)$ is the spatial Fourier transform of the envelope of the compressed waveform. The wave number value, $k_{0}$, where the value of the MTF curve is 0.1 , is used to determine the axial resolution of the imaging system. The axial resolution is defined as

$$
\lambda=1 / 2 \cdot(2 \pi) / k_{0} .
$$

2) Contrast-to-noise ratio (CNR): CNR [6], also known as contrast-to-speckle ratio, is a common quantitative measure that can assess image quality and describe the ability to perceive a target from the background region. CNR is defined as

$$
C N R=\left|\frac{\mu_{B}-\mu_{T}}{\sqrt{\sigma_{B}+\sigma_{T}}}\right|,
$$

where $\mu_{B}$ and $\mu_{T}$ are the mean intensity of the background and the target lesion, and $\sigma_{B}^{2}$ and $\sigma_{T}^{2}$ are the variance of the background and target, respectively. To avoid possible errors in the calculations due to attenuation, the evaluated regions of interest in the background and the target lesion were the same size and were located at the same depth. 


\section{RESUlTS AND Discussion}

The B-mode images for the simulated phantom under the low noise scenario are shown in Fig. 1 along with an axial profile through the center of the B-mode images. Compared to $\mathrm{CP}$, the B-mode images for REC constructed using a nonSVWF resulted in improved axial resolution but increases in sidelobe levels and deterioration in lateral resolution as depth increases. For REC-SVWF, the sidelobe levels decreased when compared to REC echoes compressed with a conventional Wiener filter. In the low noise scenario, the $\gamma$ parameter for the non-SVWF and SVWF was set to the same value.

The spatial resolutions as determined by MTF for CP and for REC echoes compressed with a conventional Wiener filter and a spatially varying Wiener filter are listed in Table I. Overall, the SVWF had a slight degradation in axial resolution in order to improve the sidelobe levels and the eSNR. Furthermore, the predicted amplitudes of the echoes due to depth dependent attenuation from each target were more correct with the SVWF than with a non-SVWF. For the non-SVWF both the signal and the noise floor were boosted. The purpose of evaluating this phantom in a low noise environment was to show that no artifacts or significant degradation in image quality occurred.

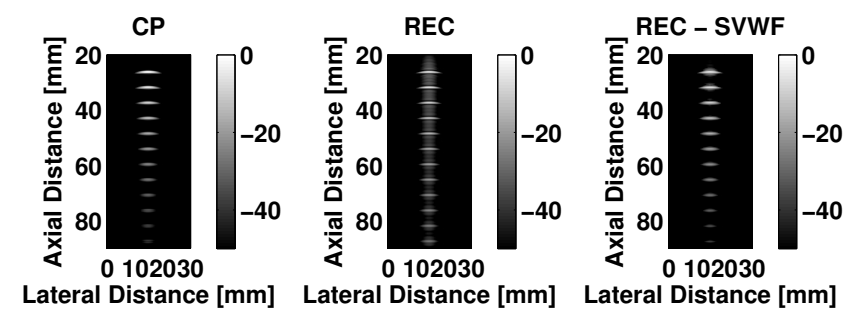

(a)

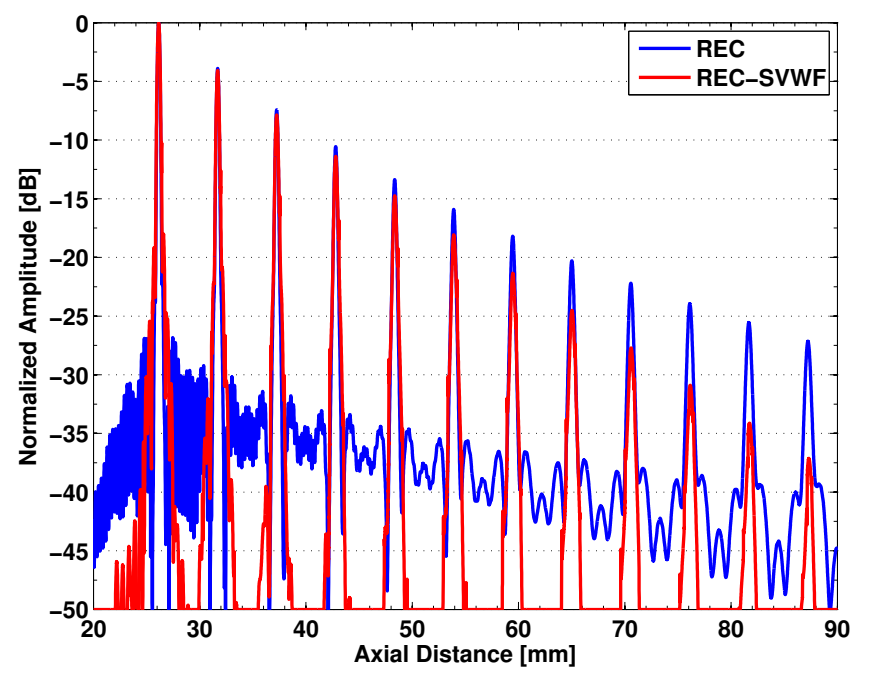

(b)

Fig. 1. (a) B-mode image and (b) on-axis plot of a simulated phantom containing 12 point targets for CP, REC and REC-SVWF for a low noise scenario.
TABLE I

SPATIAL RESOLUTION VALUES DETERMINED FROM THE MTF FOR THE SIMULATED PHANTOM - LOW NOISE SCENARIO.

\begin{tabular}{c|c|c|c}
\hline \hline Target \# & $\begin{array}{c}\text { CP } \\
(\mu m)\end{array}$ & $\begin{array}{c}\text { REC } \\
(\mu m)\end{array}$ & $\begin{array}{c}\text { REC-SVWF } \\
(\mu m)\end{array}$ \\
\hline \hline 1 & 658.1 & 368.9 & 392.6 \\
2 & 667.5 & 401.7 & 424.8 \\
3 & 687.1 & 442.2 & 458.1 \\
4 & 718.8 & 491.8 & 502.4 \\
5 & 876.1 & 554.0 & 543.3 \\
6 & 824.5 & 623.0 & 581.6 \\
7 & 910.2 & 690.5 & 591.5 \\
8 & 1015.8 & 753.6 & 922.1 \\
9 & 1121.4 & 839.7 & 973.4 \\
10 & 1274.3 & 922.2 & 1070.0 \\
11 & 1430.3 & 1008.5 & 1240.5 \\
12 & 1592.8 & 1103.7 & 1103.7 \\
\hline \hline
\end{tabular}

The B-mode images for the simulated phantom under the high noise scenario are shown in Fig. 2 along with an axial profile through the center of the B-mode images. In the high noise scenario, the SVWF allowed the operating point of the Wiener filter to remain at the same operating point as in the low-noise scenario. Consequently, when using the SVWF the axial resolution was nearly the same as in the low noise scenario with the added benefit that the noise floor was pushed below the dynamic range of the image. In the scenario where a non-SVWF was used for compression in the high noise case, the $\gamma$ parameter was adjusted until the noise was removed. This had the effect of pushing the operating point of the Weiner filter towards a matched filter. This adjustment increased sidelobe levels and degraded the axial resolution in order to improve the eSNR. Therefore, the benefits of the SVWF were clearly observed in this case.

The spatial resolutions as determined by MTF for CP and for REC echoes compressed with a non-SVWF and a SVWF are listed in Table II. Similar to the low noise case, the SVWF had a slight degradation in axial resolution in order to improve the sidelobe levels and the eSNR. In fact, an increase in eSNR of $235 \%$ was obtained over the non-SVWF at depths that typically could not be imaged using $\mathrm{CP}$ excitation. Overall, the results for simulated phantom suggested that the SVWF improved the image quality over CP and REC echoes compressed using with a non-SVWF.

Finally, the ATS 539 tissue mimicking-phantom anechoic targets were evaluated experimentally under the high noise scenario as shown in Fig. 3. An anechoic target is equivalent to a fluid-filled target with no internal scattering objects. Therefore, the only backscattered signal present in that region would be additive noise. By applying a SVWF, the noise in the anechoic target could be removed by shifting the operating point of the Wiener filter towards a matched filter. With a non-SVWF the anechoic target is smeared by either sidelobes or noise. Consequently, the actual boundaries of the anechoic target become harder to identify. This could potentially be a problem if the anechoic region was small because the sidelobes and noise could mask the anechoic target making it imperceptible. Therefore, the results of the SVWF for this 
TABLE II

SPATIAL RESOLUTION VALUES DETERMINED FROM THE MTF FOR THE SIMULATED PHANTOM - HIGH NOISE SCENARIO

\begin{tabular}{c|c|c|c}
\hline \hline Target \# & $\begin{array}{c}\text { CP } \\
(\mu m)\end{array}$ & $\begin{array}{c}\text { REC } \\
(\mu m)\end{array}$ & $\begin{array}{c}\text { REC-SVWF } \\
(\mu m)\end{array}$ \\
\hline \hline 1 & 658.1 & 379.8 & 388.3 \\
2 & 667.5 & 407.5 & 422.2 \\
3 & 687.1 & 443.6 & 461.1 \\
4 & 718.8 & 537.1 & 526.9 \\
5 & 876.1 & 631.4 & 556.2 \\
6 & 824.5 & 697.3 & 622.9 \\
7 & 910.2 & 697.4 & 640.1 \\
8 & 1015.8 & 805.6 & 922.1 \\
9 & 1121.4 & 1112.5 & 881.6 \\
10 & 1274.3 & 960.1 & 1062.0 \\
11 & 1430.3 & 1649.1 & 1158.5 \\
12 & 1592.8 & 5607.0 & 1103.7 \\
\hline \hline
\end{tabular}

target clearly displayed the benefit of using a spatially varying Wiener filter. To quantify the improvement obtained by using the SVWF, the CNR metric was applied to the two 8-mm targets located on the left of Fig. 3 and at different axial depths. The CNR for 8-mm target located at a depth of approximately $57 \mathrm{~mm}$ was estimated at $0.88,1.64,2.86$ for $\mathrm{CP}$, REC compressed with a non-SVWF, and REC compressed

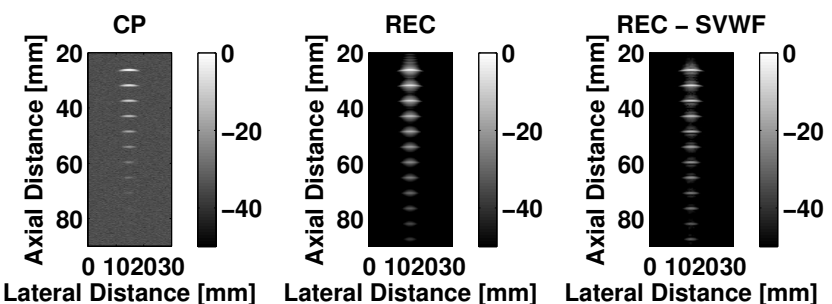

(a)

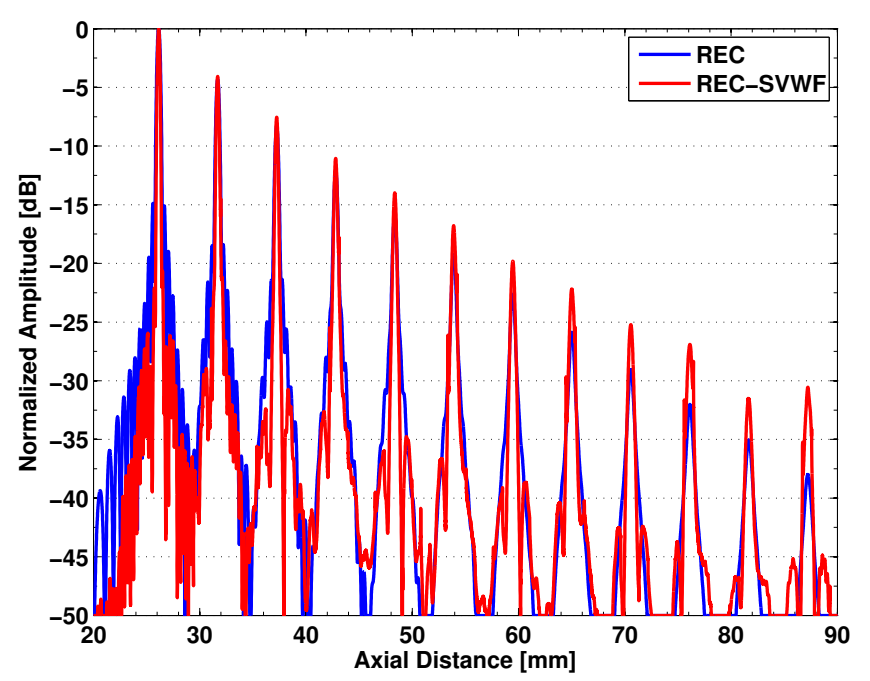

(b)

Fig. 2. (a) B-mode image and (b) on-axis plot of a simulated phantom containing 12 wire targets for CP, REC and REC-SVWF for a high noise scenario.
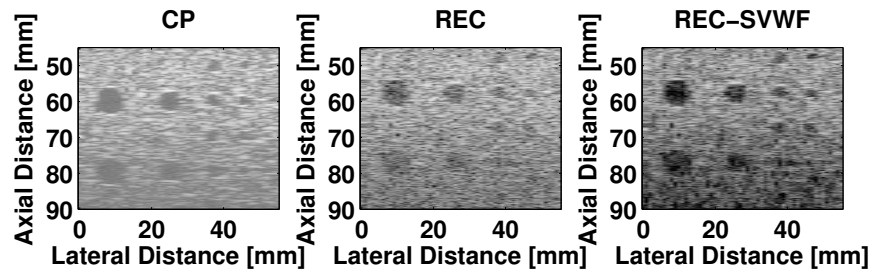

Fig. 3. Experimental results from ATS 539 tissue-mimicking phantom for CP, REC, and REC-SVWF for a high noise scenario.

with a SVWF, respectively. The CNR for 8-mm target located at a depth of approximately $77-\mathrm{mm}$ was estimated at 0.38 , $0.80,1.36$ for CP, REC compressed with a non-SVWF, and REC compressed with a SVWF, respectively. These results suggest that the SVWF based on spatially varying maps of the signal-to-noise ratio significantly improved image quality by increasing the contrast when compared to $\mathrm{CP}$ and REC echoes compressed with a conventional Wiener filter. Also, it is important to note that the SVWF removed the noise in the deeper regions of the image that appeared as speckle in the $\mathrm{CP}$ and REC non-SVWF cases.

\section{CONCLUSION}

A spatially varying Wiener filter that corrected for $\overline{e S N R}$ locally to improve compression performance was evaluated. Simulation results of point targets indicated that the eSNR was increased in deeper areas imaged at the expense of a small degradation in spatial resolution and slight increase in sidelobe levels. Furthermore, experimental measurements of anechoic targets resulted in improved image quality compared to conventional methods. Future work will evaluate techniques to optimize the SVWF.

\section{ACKNOWLEDGMENT}

This work was partially supported by NIH grant R21 EB006741.

\section{REFERENCES}

[1] T. Misaridis and J. A. Jensen, "Use of modulated excitation signals in medical ultrasound. Part I: Basic concepts and expected benefits," IEEE Trans. Ultrason. Ferroelectr. Freq. Contr., vol. 52, pp. 177-191, Feb. 2005.

[2] J. K. Tsou, J. Liu, and M. F. Insana, "Modeling and phantom studies of ultrasonic wall shear rate measurements using coded excitation, IEEE Trans. Ultrason. Ferroelectr. Freq. Contr., vol. 53, pp. 724734, Apr. 2006.

[3] M. L. Oelze, "Bandwidth and resolution enhancement through pulse compression," IEEE Trans. Ultrason. Ferroelectr. Freq. Control, vol. 54, pp. 768-781, Apr. 2007.

[4] J. A. Jensen, "A model for the propagation and scattering of ultrasound tissue," J. Acoust. Soc. Am., vol. 89, pp. 182-190, Jan. 1991.

[5] J. Beutel, H. L. Kundel, and R. L. Van Metter, Handbook of Medical Imaging. Bellingham, WA: SPIE Press, 2000.

[6] M. S. Patterson and F. S. Foster, "The improvement and quantitative assessment of B-mode images produced by an annular array/cone hybrid," Utrason. Imag., vol. 5, pp. 195-213, 1983. 